\title{
L'empreinte spatiale des approvisionnements alimentaires locaux : un modèle graphique
}

Spatial footprint of local food supplies: a graphic model

La huella ecológica del suministro local de alimentos: un modelo gráfico

Adrien Baysse-Lainé

\section{(2) OpenEdition}

Journals

Édition électronique

URL : http://journals.openedition.org/mappemonde/576

DOI : $10.4000 /$ mappemonde.576

ISSN : 1769-7298

Éditeur

UMR ESPACE

Référence électronique

Adrien Baysse-Lainé, «L'empreinte spatiale des approvisionnements alimentaires locaux : un modèle graphique », Mappemonde [En ligne], 122 | 2017, mis en ligne le 08 décembre 2017, consulté le 20 juin 2019. URL : http://journals.openedition.org/mappemonde/576 ; DOI : 10.4000/mappemonde.576

Ce document a été généré automatiquement le 20 juin 2019

\section{cc) (†) ()}

La revue Mappemonde est mise à disposition selon les termes de la Licence Creative Commons Attribution - Pas d'Utilisation Commerciale - Partage dans les Mêmes Conditions 4.0 International. 


\section{L'empreinte spatiale des approvisionnements alimentaires locaux : un modèle graphique}

Spatial footprint of local food supplies: a graphic model

La huella ecológica del suministro local de alimentos: un modelo gráfico

Adrien Baysse-Lainé

\section{Introduction}

1 Le renouveau des circuits alimentaires de proximité (Praly et al., 2014) s'appuie notamment sur l'idée d'un renforcement de la proximité géographique entre les producteurs, d'éventuels intermédiaires et les consommateurs. Cette dimension spatiale a cependant fait l'objet de peu de travaux, que l'on peut regrouper autour de trois thématiques: la localisation des points de vente des circuits courts (Scheffer, Dalido, 2010 ; Schneller, 2010) et des activités liées à l'alimentation locale (Ilbery et al., 2006) ; les flux engendrés par les circuits de proximité (Penker, 2006); la délimitation de bassins d'approvisionnement qui rendraient des régions urbaines autonomes en alimentation (Peters et al., 2009; Darrot, 2012; Corsi et al., 2015). Mais les liens entre l'approvisionnement alimentaire local tel qu'il existe et les formes spatiales des activités de production et de transformation agricoles restent largement inexplorés (Capt et al., 2014, p. 105). Nous proposons d'étudier ici la traduction dans l'espace des relations alimentaires de proximité entre villes et campagnes, c'est-à-dire la structure spatiale de l'agriculture qui « nourrit » localement la ville.

2 Pour ce faire, nous prenons comme objet l'empreinte spatiale de l'alimentation locale urbaine, que nous définissons comme l'ensemble des terres agricoles participant à nourrir les citadins par des filières locales (courtes ou longues) - de manière directe (par la production d'aliments) ou indirecte (par la production d'intrants agricoles utilisés pour produire ces aliments). L'écologie territoriale a déjà dressé des portraits diachroniques de 
l'empreinte environnementale (Barles, 2008) - notamment alimentaire - de grandes métropoles (Billen et al., 2012). Nous cherchons ici à mettre en œuvre une approche restreinte à l'alimentation locale et adaptée à des villes de tailles plus réduites. Alors que les travaux de l'écologie territoriale documentent précisément certains cas (comme l'approvisionnement alimentaire de Brive [Marty, 2013] ou celui de Paris en produits laitiers [Chatzimpiros, 2011]), notre ambition est de concevoir un modèle généralisable de l'empreinte alimentaire locale d'une ville. Il s'agit d'examiner selon quels critères et à l'aide de quelles formes spatiales il est possible de modéliser graphiquement la composante locale de cette empreinte. Notre hypothèse est que la distance géographique ne suffit pas à élaborer un tel modèle, qui doit intégrer la diversité des inscriptions spatiales des circuits locaux et la variété des définitions du « local ».

Après une présentation du cadre méthodologique, l'article met en évidence que certains modèles spatiaux mobilisés pour penser la structure spatiale des approvisionnements alimentaires locaux contemporains sont dépassés. Il propose alors de bâtir un modèle graphique alternatif, qui corresponde mieux aux nouvelles pratiques d'approvisionnement local, tant du point de vue des consommateurs que des producteurs et des intermédiaires. Enfin, pour en évaluer la pertinence, ce modèle est testé avec des données collectées à Millau.

\section{Terrain et méthodologie}

4 La première partie de l'étude a pour objectif la proposition d'un double modèle graphique. Nous l'avons construit à partir de critères sélectionnés dans vingt-cinq publications anglophones et francophones relatives aux définitions spatiales du « local» dans les circuits courts ainsi qu'aux formes et localisations des aires d'approvisionnement des circuits longs.

5 Les résultats que nous présentons ensuite pour tester le modèle sont issus d'une enquête de terrain menée en 2014 à Millau et dans sa périphérie (principalement les causses et vallées du Sud-Aveyron). La ville est de taille restreinte : elle se limite à une unité urbaine partagée entre deux communes (aux territoires majoritairement non bâtis) comptant 23 000 habitants. Cette petite taille a permis de recenser la quasi-totalité des circuits locaux, dans des commerces de détail, des grandes surfaces, des restaurants collectifs, des lieux de vente directe (marché, magasin de producteurs, distributions de paniers). Face à l'absence d'une définition établie du « local», nous avons pris en compte tous les circuits inscrits dans un rayon d'une centaine de kilomètres autour de la ville, correspondant peu ou prou aux territoires de cinq départements (Aveyron, Lozère, Gard, Hérault, Tarn) au milieu desquels se situe Millau.

6 Cette enquête s'est déroulée en trois étapes (tableau 1), qui ont permis de dresser la liste des circuits alimentaires locaux puis de quantifier et localiser les surfaces utilisées pour les approvisionner. Nous avons d'abord recensé tous les circuits de vente directe, par une recherche sur Internet et en discutant de leurs pratiques d'achat avec des habitants. Les producteurs de ces circuits ont été rencontrés dans les lieux de vente, ils nous ont fourni des renseignements sur les surfaces cultivées pour approvisionner Millau. Nous avons ensuite relevé les provenances des aliments dans les magasins de la ville, à l'aide de l'adresse du site de production ou du code emballeur ${ }^{1}$. Des entretiens avec les commerçants et les restaurateurs collectifs ont permis de compléter cette liste de fournisseurs locaux (agriculteurs, transformateurs et grossistes), notamment pour les 
approvisionnements saisonniers. Nous avons enfin joint par téléphone ces fournisseurs : les agriculteurs (cas de circuits courts à un intermédiaire) nous ont fourni des renseignements sur les surfaces cultivées, et les transformateurs et grossistes (cas des circuits longs) des renseignements sur leurs aires d'approvisionnement.

7 Nous avons ainsi recensé 118 exploitations agricoles en circuit court, dont nous connaissons la localisation à l'échelle communale (pour l'ensemble des exploitations) et les surfaces précises qu'elles utilisent pour approvisionner Millau (pour $74 \%$ des exploitations). Nous avons également reconstitué les aires d'approvisionnement de 59 intermédiaires de filières longues locales. Ces circuits alimentaires sont diversifiés, malgré une forte représentation des produits carnés et laitiers, liée à la spécialisation en élevage de la région. Pour les circuits courts, les quatre productions les plus répandues sont les produits carnés (produits par $37 \%$ des exploitations), les légumes ( $26 \%$ ), les fruits $(16 \%)$ et les produits laitiers $(12 \%)$. Pour les circuits longs, il s'agit des produits carnés (commercialisés par $34 \%$ des intermédiaires), des produits laitiers $(25 \%)$, des céréales, de la farine et du pain (19\%) et des plats cuisinés (12\%). Deux éléments participent à expliquer cette diversité: les nombreux retours à la terre qui ont accompagné et suivi la lutte du Larzac ont souvent pris la forme d'exploitations qui ont précocement privilégié la commercialisation locale; plus récemment, la Chambre d'agriculture note que depuis le milieu des années 2000, le tiers des installations dans le Sud-Aveyron intègrent au moins un atelier en circuit court.

Tableau 1. Démarche méthodologique

\begin{tabular}{|c|c|c|c|}
\hline & Étape & Modalités et lieux d'enquêtes & Population d'enquête \\
\hline 1. & $\begin{array}{l}\text { Recensement et rencontre } \\
\text { des producteurs en vente } \\
\text { directe locale. }\end{array}$ & $\begin{array}{l}\text { Recensement: Internet, discussions } \\
\text { avec les habitants. Rencontre : } \\
\text { lieux de vente directe, à Millau } \\
\text { (unité urbaine). }\end{array}$ & \multirow{3}{*}{$\begin{array}{l}118 \text { exploitations } \\
\text { agricoles recensées, dont } \\
74 \% \text { ont pu être } \\
\text { enquêtées. } \\
59 \text { intermédiaires de } \\
\text { circuits longs recensés, } \\
\text { dont } 100 \% \text { ont été } \\
\text { enquêtés. }\end{array}$} \\
\hline 2. & $\begin{array}{l}\text { Recensement } \quad \text { des } \\
\text { fournisseurs locaux des } \\
\text { magasins et } \\
\text { collectifs. }\end{array}$ & $\begin{array}{l}\text { Relevés de provenance en magasin, } \\
\text { entretiens avec commerçants et } \\
\text { restaurateurs, à Millau (unité } \\
\text { urbaine). }\end{array}$ & \\
\hline 3. & $\begin{array}{l}\text { Enquête téléphonique et } \\
\text { questionnaires auprès des } \\
\text { fournisseurs. }\end{array}$ & $\begin{array}{l}\text { Questionnaires: } \text { agriculteurs les } \\
\text { plus proches de la ville. Appels } \\
\text { téléphoniques: } \\
\text { fournisseurs. }\end{array}$ & \\
\hline
\end{tabular}

\section{Les modèles graphiques agricoles des $\mathrm{XIX}^{\mathrm{e}}$ et $\mathrm{XX}$ siècles et l'alimentation locale contemporaine}

Aucun modèle graphique n'est strictement dédié à l'approvisionnement alimentaire. Ce sont plutôt les utilisations agricoles des sols autour des villes et villages qui ont été étudiées, aux XIX et $\mathrm{XX}^{\mathrm{e}}$ siècles. Le modèle de Von Thünen (1826) décrit ainsi la structure spatiale idéale-typique des activités agricoles autour d'une ville. Dans une perspective 
d'économie géographique, où les coûts de transport (proportionnels à la distance) expliquent des différentiels de rente entre productions, six cercles concentriques spécialisés entourent la ville. Se succèdent ainsi du maraîchage, de l'horticulture et de l'élevage laitier; de la sylviculture ; des grandes cultures; une alternance de cultures et de pâturages; un assolement triennal à jachère; de l'élevage extensif. L'approvisionnement alimentaire de la ville - seule agglomération de sa région constitue de fait un objet de la modélisation, puisque tous les produits de l'agriculture sont censés y être commercialisés.

D'autres modèles ont été ultérieurement élaborés par des géographes pour décrire la structure spatiale des activités agricoles à une échelle plus fine, celle du finage villageois traditionnel. Le modèle graphique le plus simplifié de ce type prend ainsi la forme de trois auréoles (jardins/champs/pâturages) correspondant à une intensité décroissante de l'utilisation du sol (Lienau, 1995, p. 39). W. Müller-Wille (1955) et A. Krenzlin (1962) parmi d'autres - ont proposé des modèles graphiques plus complexes, en ajoutant aux auréoles des zones en parts de camembert. H. Becker (1998, p. 190) en propose une application en délimitant au sein d'un territoire communal idéal-typique quatre cercles concentriques (jardins/champs privés/champs communaux/pâturages et forêts communaux), au sein desquels des zones de prairies et de jardins se découpent en parts de camemberts tronquées. Les flux alimentaires sont encore laissés de côté : la production agricole est destinée à une commercialisation principalement locale.

Certaines représentations contemporaines de ce que devrait être l'inscription spatiale d'une alimentation urbaine «relocalisée »entretiennent des ressemblances avec ces modèles graphiques : comme le montrent les trois exemples suivants, on y retrouve des auréoles concentriques centrées sur la ville et spécialisées. L'organisation communautaire «Growing Communities » imagine comment Londres pourrait être nourrie de manière plus locale, à partir d'un bassin d'approvisionnement modélisé en sept auréoles concentriques. À chacune de ces auréoles sont attribués un volume et des types de productions. Les trois premières (agriculture urbaine et périurbaine) seraient affectées à la production de fruits et légumes, pour $25 \%$ de l'alimentation de la ville, tandis que la quatrième (arrière-pays rural dans un rayon de 100 miles $-161 \mathrm{~km}$ ) fournirait $35 \%$ des besoins alimentaires, à partir de grandes cultures et d'élevage ${ }^{2}$. Le projet de recherche européen "Foodmetres" partage cette vision prospective d'un approvisionnement alimentaire local de la ville en auréoles, ouvertement inspirée du modèle graphique de Von Thünen (Wascher et al., 2015, p. 38-45) : une première auréole serait dédiée aux cultures et une deuxième à l'élevage. Enfin, en France, le modèle de la ceinture maraîchère périurbaine est promu par le mouvement des "Colibris " pour relocaliser l'alimentation ${ }^{3}$, en référence à des systèmes vivriers disparus au cours du $\mathrm{XX}^{\mathrm{e}}$ siècle (Poulot, 2010). Quand des acteurs publics cherchent à renforcer la dimension nourricière de l'agriculture de proximité dans leur territoire, c'est encore cette forme spatiale auréolaire centrée sur la ville (« ceinture périurbaine ») qui est mentionnée, par exemple à Rodez ${ }^{4}$ ou à Paris 5 .

11 Les modèles graphiques des siècles passés apparaissent en filigrane dans ces représentations contemporaines. Ils s'appliquent toutefois originellement à un contexte rural, antérieur à la révolution des transports et à la modernisation agricole - qui ont participé à spécialiser les orientations productives de régions entières. Les distances parcourues par les aliments entre lieux de production et lieux de consommation ont fortement augmenté depuis deux siècles (Billen et al., 2012), de même que le nombre 
d'intermédiaires par lesquels ils transitent. Ces modèles graphiques ne sont donc plus adaptés pour analyser la spatialité de l'alimentation urbaine. Il s'agit de forger de nouveaux outils pour appréhender les transformations issues du regain d'intérêt des consommateurs, des producteurs, des distributeurs et des pouvoirs publics pour les circuits alimentaires locaux.

\section{La modélisation graphique au service d'un nouveau regard sur les approvisionnements alimentaires locaux urbains}

12 Afin de cerner les contours de l'empreinte spatiale de l'alimentation locale urbaine, nous définissons un cadre d'analyse de l'approvisionnement alimentaire puis en ébauchons un modèle graphique en deux parties, à partir d'une étude de la littérature centrée sur les critères de définition $\mathrm{du}$ « local ».

\section{Les trois secteurs spatiaux de l'empreinte alimentaire locale d'une ville}

13 Le point de départ de notre cadre d'analyse est la demande urbaine d'alimentation locale. Quand on cherche à reconstituer les trajets parcourus par les aliments depuis les parcelles jusqu'aux consommateurs, cette demande peut être scindée en deux. D'un côté, les produits vendus en circuit court ont une origine relativement simple à identifier. De l'autre côté, les produits transitant par au moins deux intermédiaires, parfois transformés et mélangés à d'autres, ont une origine plus difficile à établir. La collecte des données se rapportant à ces deux types de circuits suit des protocoles différents, présentés dans le tableau 1 ; par ailleurs, les dynamiques des circuits courts et longs diffèrent : nous considérons donc que les terres agricoles intégrées à ces deux types de filières relèvent de deux secteurs différents de l'empreinte spatiale. Un dernier secteur répond à notre définition étendue de l'approvisionnement alimentaire : il rassemble les terres cultivées ou pâturées (toujours à proximité de la ville, dans un rayon d'une centaine de kilomètres) pour la production d'intrants utilisés par les exploitations des deux autres secteurs (figure 1). Notre étude est décomposée ci-dessous secteur par secteur ; faute de données suffisantes pour le troisième secteur, nous nous limiterons aux deux premiers. 
Figure 1. Les trois secteurs du bassin d'approvisionnement alimentaire local d'une ville

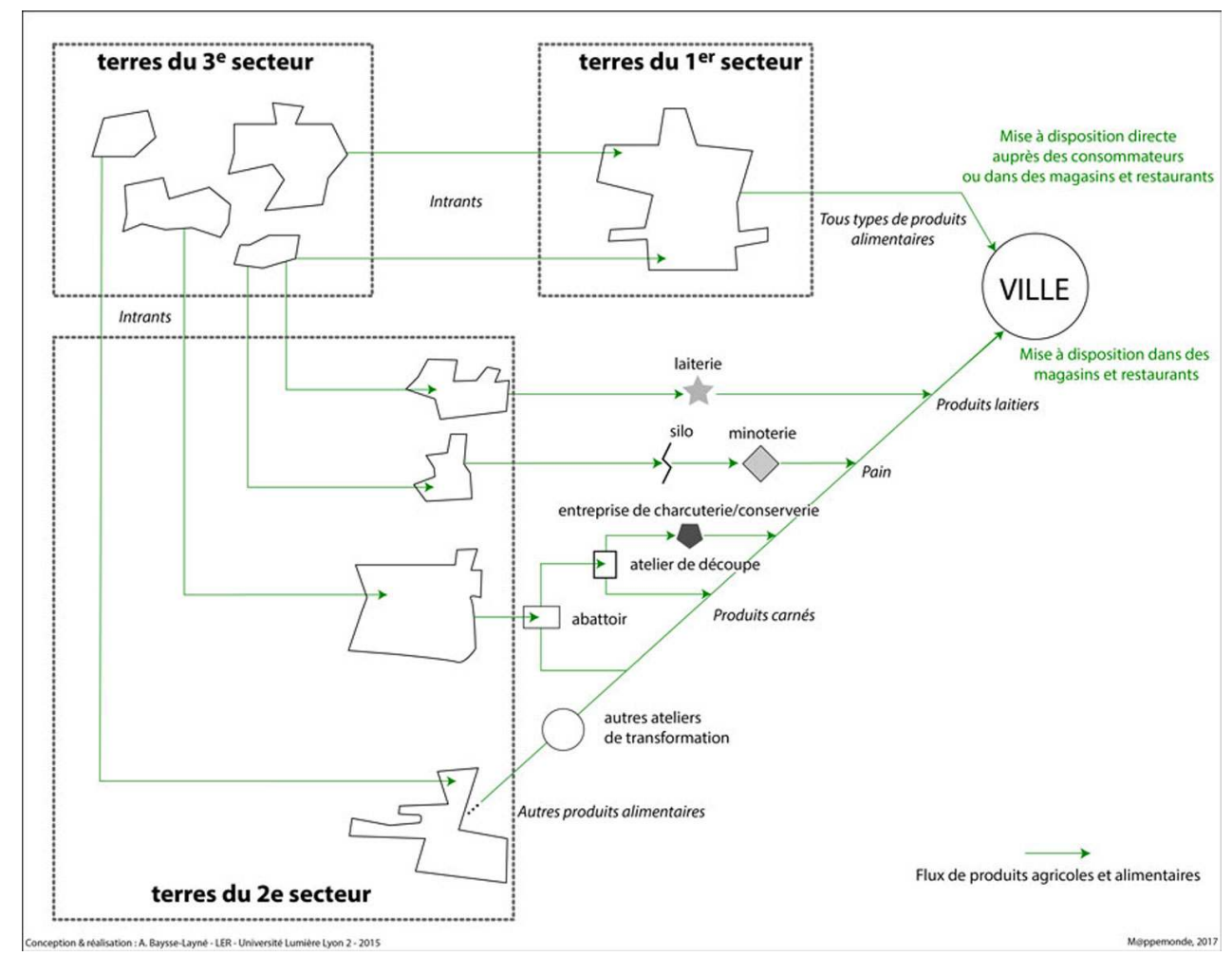

Conception \& réalisation : A. Baysse-Layné, LER - Université Lumière Lyon 2 - 2015 localisation précise de chacune des parcelles approvisionnant la ville : nous cherchons plutôt à cerner la densité de ces parcelles. C'est que la localisation précise des parcelles dépend moins du mode de commercialisation que de facteurs relatifs à l'accès à la terre souvent liés à des décisions individuelles ou conjoncturelles, difficilement modélisables. De ce fait, elle n'entretient pas de lien particulier avec le côté local ou non de l'approvisionnement. Par contre, si l'on s'intéresse à la part de ces parcelles dans la surface agricole totale - c'est-à-dire à la densité de l'empreinte spatiale de l'alimentation locale - le lien est plus clair. En effet, les flux alimentaires locaux proviennent par définition de zones proches de la ville qui les commande, sans distinction entre les parcelles incluses dans ce périmètre, chacune étant aussi « locale » que les autres. Étudier l'empreinte au niveau agrégé de sa densité permet de rendre compte de cette spécificité.

\section{L'empreinte spatiale des circuits courts : un gradient centre- périphérie déformé par les représentations et pratiques du « local »}

Les terres du premier secteur sont à la base d'un approvisionnement volontairement local, tant pour les consommateurs que pour les producteurs. Ce sont donc les définitions de ce «local» qui président au modelage de la structure spatiale de ce secteur, qu'elles relèvent d'un «localisme contemporain » (le «locavorisme») ou «traditionnel» (des achats locaux guidés par l'habitude ou l'envie de produits frais et/ou bon marché) (McEntee, 2010). Ces définitions partagent un principe de minimisation de la distance entre les lieux de production (voire de transformation) et de consommation. D'après une 
récente revue de littérature (Feldmann et Hamm, 2015), cette distance constitue le principal critère du caractère "local» d'un aliment. De vastes enquêtes par questionnaires menées aux États-Unis (Selfa et Qazi, 2005 ; Ostrom, 2006 ; Hu et al., 2010 ; Adams et Adams, 2011) et en Europe (Khan et Prior, 2010 ; Carroll et Fahy, 2014) ont cherché à évaluer sa part face à d'autres critères. Elle varie toutefois de $12 \%$ à $91 \%$, du fait d'écarts dans les formulations des questions et de contextes variés (les formes de villes et de mobilités diffèrent largement entre les États-Unis et l'Europe). Ces travaux soulignent l'absence d'une limite précise du « local »: la distance est un critère de choix relatif plus qu'une caractéristique absolue du «local» (Futamara, 2007). À l'opposé de formalisations figées (une distance de 100 miles $-161 \mathrm{~km}$ - étant par exemple souvent évoquée pour les États-Unis; ou de 30 miles - $48 \mathrm{~km}$ - au Royaume-Uni, par l'association nationale des marchés de producteurs), ces enquêtes renvoient à une palette de distances. À titre illustratif, citons une enquête réalisée dans l'Ohio (Hu et al., 2010): la distance maximale entre producteur et consommateur pour qu'un produit soit considéré comme local est de $40 \mathrm{~km}$ pour $100 \%$ des sondés, $80 \mathrm{~km}$ pour $46 \%, 121 \mathrm{~km}$ pour $23 \%, 161 \mathrm{~km}$ pour $17 \%$ et $322 \mathrm{~km}$ pour $3 \%$. Notre modèle recourt en conséquence à un gradient centre-périphérie autour de la ville pour rendre compte de ce rôle de la distance (figure 2 ).

Figure 2. Un modèle graphique du premier secteur

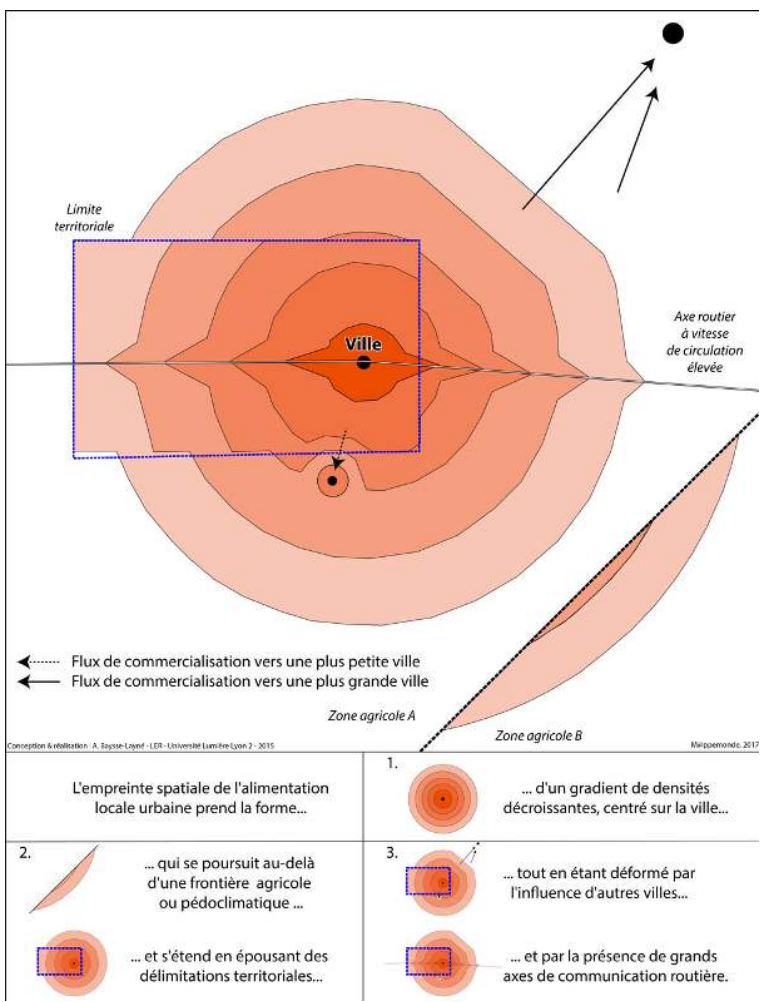

Conception \& réalisation : A. Baysse-Layné, LER - Université Lumière Lyon 2 - 2015

Deux ajustements de ce gradient découlent du comportement des consommateurs. Tout d'abord, les échelles du « local » varient en fonction des aliments : M. Ostrom (2006, p. 69) rapporte que pour certains enquêtés, des fruits et légumes sont considérés comme locaux uniquement s'ils sont issus d'une zone restreinte (la vallée), tandis que le reste des aliments l'est même s'il vient d'un périmètre plus étendu (l'État). D'après B. Carroll et 
F. Fahy (2014, p. 8-9), cette différenciation est liée aux différentiels de disponibilité : si un aliment peut être produit à proximité, il n'est considéré comme local que s'il en provient ; à l'inverse, un produit absent de la zone initialement définie comme locale, mais commun dans une zone plus éloignée est considéré comme local à l'aune de critères étendus. Il peut s'agir par exemple de viande dans une région de grandes cultures (Poulot, 2014, p. 48) ou de produit "bio » dans une région comptant peu d'agriculteurs biologiques labellisés (Marty, 2013, p. 509-512). Étant le plus local possible, un tel approvisionnement est assimilé à la catégorie «local ». Une composante discontinue s'ajoute ainsi à notre représentation continue de l'empreinte spatiale de l'alimentation locale. Elle est représentée dans le modèle graphique (figure 2) par un isolat du gradient au-delà d'une frontière.

Les délimitations du « local » ne s'appuient pas seulement sur la distance : elles intègrent aussi une composante territoriale. Cette conception renvoie à trois types de produits: d'abord les produits de terroir, identifiés à une origine territoriale et qualifiant le territoire en retour (Delfosse, 2013), ensuite les produits locaux " sans nom », consommés frais et liés au quotidien (Delfosse, 2012), enfin les " produits ordinaires requalifiés », à la croisée des deux premiers types (Garçon, 2012). Divers éléments facilitent la reconnaissance du caractère local de ces produits par les consommateurs (alors que le calcul d'une distance est plus complexe) : un nom, un label ou une marque territoriale ${ }^{6}$ pour les produits de terroir et les produits ordinaires requalifiés, une adresse ou un code postal pour les produits "sans nom». Les découpages, notamment institutionnels, constituent ainsi des catégories souvent mobilisées par les consommateurs pour définir le « local » (Wilkins et al., 1996; Gallons et al., 1997 ; Brown, 2003). La mise en regard des principales études sur le sujet (tableau 2) rend compte de l'emboîtement des échelles territoriales mobilisées par les consommateurs. Le niveau considéré comme local par plus de la moitié des enquêtés est soit le comté et des comtés voisins (dans le cas d'États étendus), soit l'État ou le pays (dans le cas de petits États et de l'Irlande). À notre conception en gradient de l'empreinte spatiale de l'alimentation locale s'ajoute donc une composante territoriale. Elle est représentée dans le modèle (figure 2) par une déformation positive du gradient centre-périphérie à l'intérieur des limites d'une entité spatiale.

Tableau 2. Définitions du « local » en fonction de découpages territoriaux

\begin{tabular}{|c|c|c|c|c|c|c|c|}
\hline \multicolumn{8}{|c|}{ Part des enquêtés pour lesquels un produit est local... } \\
\hline $\begin{array}{l}\text { Wilkins et } \\
\text { al., } 1996 \\
\text { (nord-est } \\
\text { des États- } \\
\text { Unis) }\end{array}$ & $\begin{array}{l}\text { Gallons et } \\
\text { al., } \quad 1997 \\
\text { (Delaware) }\end{array}$ & $\begin{array}{l}\text { Brown, } \\
2003 \text { (sud- } \\
\text { est du } \\
\text { Missouri) }\end{array}$ & $\begin{array}{l}\text { Ostrom, } 2006 \\
\text { (Washington } \\
\text { [État]) }\end{array}$ & $\begin{array}{l}\mathrm{Hu} \text { et } \\
\text { al., } \\
2010 \\
\text { (Ohio) }\end{array}$ & $\begin{array}{l}\text { Adams et } \\
\text { Adams, } \\
2011 \\
\text { (Floride) }\end{array}$ & $\begin{array}{l}\text { Carroll et } \\
\text { Fahy, } \\
2014 \\
\text { (Irlande) }\end{array}$ & $\begin{array}{l}\ldots \\
\text { provient : }\end{array}$ \\
\hline- & - & - & $100 \%$ & - & - & - & $\begin{array}{l}\mathrm{du} \\
\text { voisinage }\end{array}$ \\
\hline - & - & - & $97 \%$ & - & - & - & $\begin{array}{l}\text { de la } \\
\text { commune }\end{array}$ \\
\hline
\end{tabular}




\begin{tabular}{|c|c|c|c|c|c|c|c|}
\hline $100 \%$ & - & - & - & - & - & - & $\begin{array}{l}\text { d'une } \\
\text { partie du } \\
\text { comté }\end{array}$ \\
\hline $84 \%$ & - & $100 \%$ & $79 \%$ & - & - & $100 \%$ & du comté \\
\hline $62 \%$ & - & $86 \%$ & $55 \%$ & - & - & - & $\begin{array}{l}\text { du comté et } \\
\text { de comtés } \\
\text { voisins }\end{array}$ \\
\hline- & - & $72 \%$ & - & - & - & - & $\begin{array}{l}\text { d'une } \\
\text { partie de } \\
\text { l'État }\end{array}$ \\
\hline- & - & $23 \%$ & - & - & - & - & $\begin{array}{l}\text { de parties } \\
\text { voisines de } \\
\text { plusieurs } \\
\text { États }\end{array}$ \\
\hline $46 \%$ & $83 \%$ & $12 \%$ & $25 \%$ & $11 \%$ & $9 \%$ & $69 \%$ & $\begin{array}{lr}\text { de r l'État } \\
\text { (ou r } \\
\text { pays, } \\
\text { l'Irlande) }\end{array}$ \\
\hline $15 \%$ & $\begin{array}{l}34 \% \text { à } 48 \% \\
\text { (plusieurs } \\
\text { options) }\end{array}$ & - & - & - & - & - & $\begin{array}{l}\text { de l'État et } \\
\text { de parties } \\
\text { d'États } \\
\text { voisins }\end{array}$ \\
\hline- & - & - & $3 \%$ & - & $2 \%$ & - & $\begin{array}{l}\text { d'une } \\
\text { région des } \\
\text { États-Unis }\end{array}$ \\
\hline- & - & - & $1 \%$ & $1 \%$ & $1 \%$ & $3 \%$ & $\begin{array}{l}\text { des États- } \\
\text { Unis (ou du } \\
\text { continent, } \\
\text { pour } \\
\text { l'Irlande) }\end{array}$ \\
\hline
\end{tabular}

Deux autres ajustements du gradient découlent de la manière dont les producteurs choisissent d'approvisionner localement une ville plutôt qu'une autre. La ville de notre modèle n'est en effet pas isolée au milieu d'une région agricole la nourrissant exclusivement: des centres urbains proches s'approvisionnent aussi par des circuits courts locaux. Pour intégrer cette concurrence à notre modèle, nous avons pris en compte les critères de choix des producteurs. D'après les quelques travaux ayant abordé cette question, les deux principaux critères sont la taille et le niveau de vie du marché d'un côté (Schneller, 2010 ; Chevallier et al., 2014), la facilité d'accès au lieu de vente et sa distance-temps à l'exploitation de l'autre (Schneller, 2010 ; Blanquart et Gonçalves, 2011). Le premier est représenté dans le modèle (figure 2) par des déformations négatives du gradient centre-périphérie à proximité d'autres villes, dont l'ampleur varie selon la taille 
des villes. Le deuxième se traduit par des déformations positives le long des axes où la vitesse de circulation est élevée (figure 2).

\section{L'empreinte spatiale des circuits longs : des zones d'approvisionnement sous faible influence de la ville}

Les terres du deuxième secteur sont à la base d'un approvisionnement qui est local de fait : la proximité géographique y est rarement mise en avant comme une caractéristique essentielle des produits. Beaucoup de filières longues locales le sont ainsi seulement en partie : la consommation d'un fromage AOC dans l'aire de collecte de son lait se fait par l'intermédiaire d'une filière longue (éleveur/transformateur/commerçant/ consommateur), mais locale, qui ne correspond généralement qu'à une part minime du total des ventes. La morphologie de ce secteur suit donc celle des bassins de production. La localisation des infrastructures de transformation et de conditionnement des filières agroalimentaires induit en effet en partie celle d'une zone d'approvisionnement (Corniaux et al., 2014), et in fine des terres agricoles du deuxième secteur. La structure spatiale de l'empreinte alimentaire urbaine semble alors indépendante de la localisation de la ville.

La proximité aux infrastructures, qui permet une minimisation des frais logistiques, constitue un premier critère de la morphologie des bassins de collecte (Vaudois, 2000). Deux autres types de facteurs l'influencent. La modalité de l'approvisionnement d'abord : s'il s'agit d'une collecte régulière (par exemple de lait) opérée par le transformateur, le trajet suivi - et donc l'inclusion ou non d'une exploitation à la collecte - est surtout guidé par des impératifs économiques. Lorsque la collecte a lieu rarement (par exemple pour les céréales), d'autres critères que la rationalité économique peuvent intervenir. Les zones d'approvisionnement sont en effet également façonnées par des critères historiques, sociaux et économiques. C. Petit et C. Aubry (2012) soulignent que des producteurs démarchant collectivement une infrastructure de transformation pour entrer dans sa collecte, en proposant de participer aux frais logistiques supplémentaires, peuvent être à l'origine d'une extension du bassin de collecte.

21 En termes de modélisation graphique, les zones d'approvisionnement des filières longues locales restent complexes à dessiner. Quatre principaux types ressortent néanmoins. Un gradient centre-périphérie (autour de l'infrastructure de transformation) peut correspondre à la situation d'une unité relativement nouvelle ayant défini un rayon de collecte économiquement optimal (figure 3, type 1). Ce gradient peut connaître des déformations, par exemple dues au rassemblement de plusieurs collectes vers une unité de transformation unifiée (Jénot et Desmaison, 2009). D'après la cartographie de C. Petit pour l'île-de-France (2013, p. 408-409), certaines zones d'approvisionnement ont des formes complexes, en polygones à de nombreux côtés (figure 3, type 2). Selon C. Margetic (2015, p. 38), les bassins de production des industries agroalimentaires en France sont des " réseaux complexes [associant] un dispositif en archipel [avec] un noyau central et de[s] noyaux secondaires » (figure 3, type 3). Enfin, dans une région de production spécialisée, où le maillage des unités de transformation est dense (cas des abattoirs en zone d'élevage), les zones d'approvisionnement se partagent l'espace en zones partiellement superposées (figure 3, type 4). 
Figure 3. Un modèle graphique du deuxième secteur

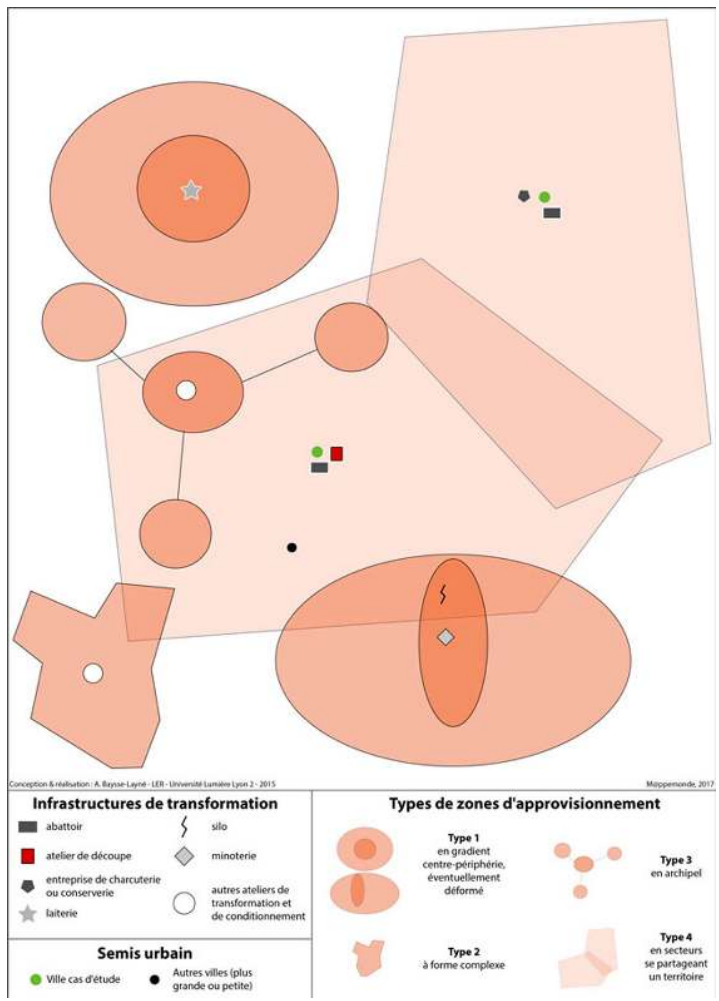

Conception \& réalisation : A. Baysse-Layné, LER - Université Lumière Lyon 2 - 2015

Notre modèle graphique de l'empreinte spatiale de l'alimentation locale urbaine s'appuie sur une conception tripartite de l'approvisionnement. Les représentations du «local», qui guident les comportements des consommateurs et des producteurs, forment la base $\mathrm{du}$ modèle du premier secteur. Le modèle du deuxième secteur rassemble plusieurs modalités de l'approvisionnement des intermédiaires agroalimentaires. Pour affiner cette ébauche, nous en proposons une application empirique.

\section{D'où se nourrit Millau localement ? Application du modèle graphique à une petite ville du Massif central}

\section{Un approvisionnement en circuits courts aveyronnais et caussenard, concurrencé par d'autres villes}

Les surfaces cultivées ou pâturées pour nourrir Millau ont été précisément chiffrées et localisées pour $74 \%$ des exploitations. Pour les $26 \%$ restants, la commune de rattachement est connue, sans évaluation des surfaces. En calculant à l'échelle communale la densité de ces terres parmi le total des terres agricoles, nous avons pu délimiter cinq zones de densités décroissantes du premier secteur. Ce gradient centrepériphérie n'est pas représenté par des cercles concentriques comme dans le modèle, mais par des ellipses aux orientations variées (figure 4), ces formes s'adaptant mieux à la distribution spatiale observée. 
Figure 4. Application au cas de Millau. Le gradient centre-périphérie du premier secteur

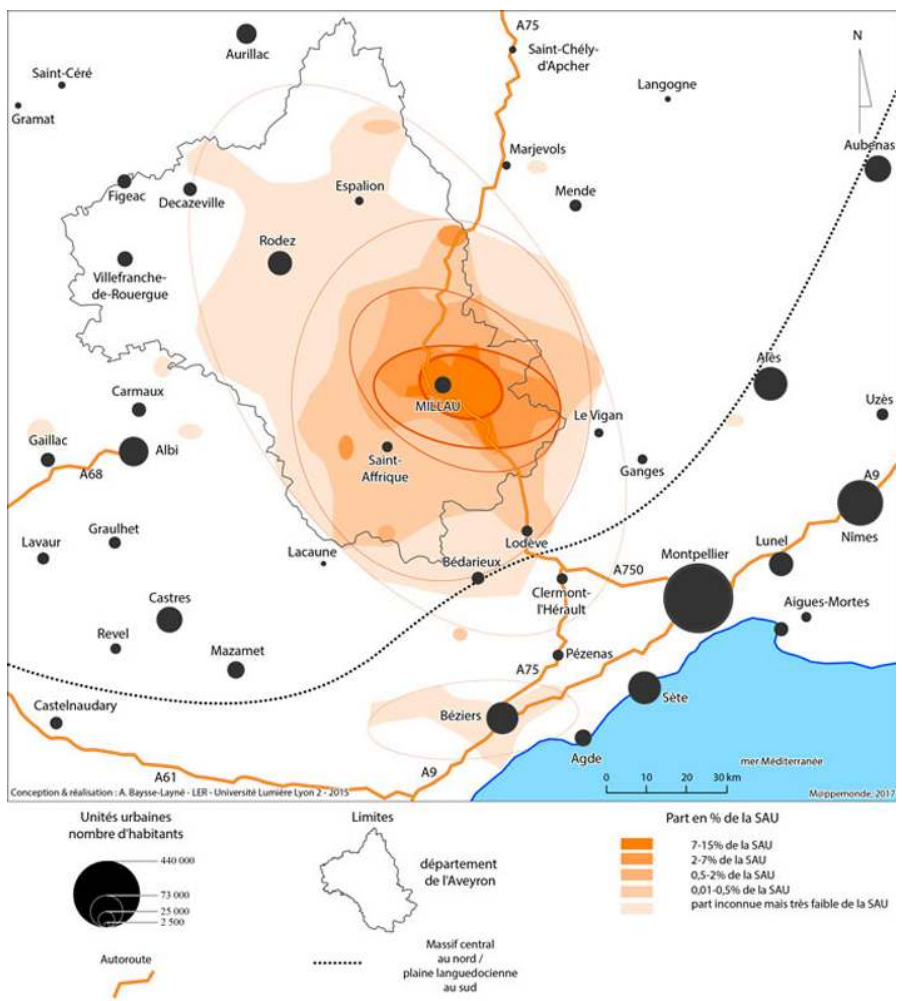

Conception \& réalisation : A. Baysse-Layné, LER - Université Lumière Lyon 2 - 2015

24 Ces ellipses correspondent aux cercles concentriques du modèle graphique : elles constituent des approximations spatiales. La figure 5 présente leurs morphologies une fois déformées pour correspondre plus finement aux densités calculées. Il s'agit d'évaluer dans quelle mesure ces contours s'expliquent par les facteurs du modèle. 
Figure 5. Application au cas de Millau. Le modèle graphique du premier secteur

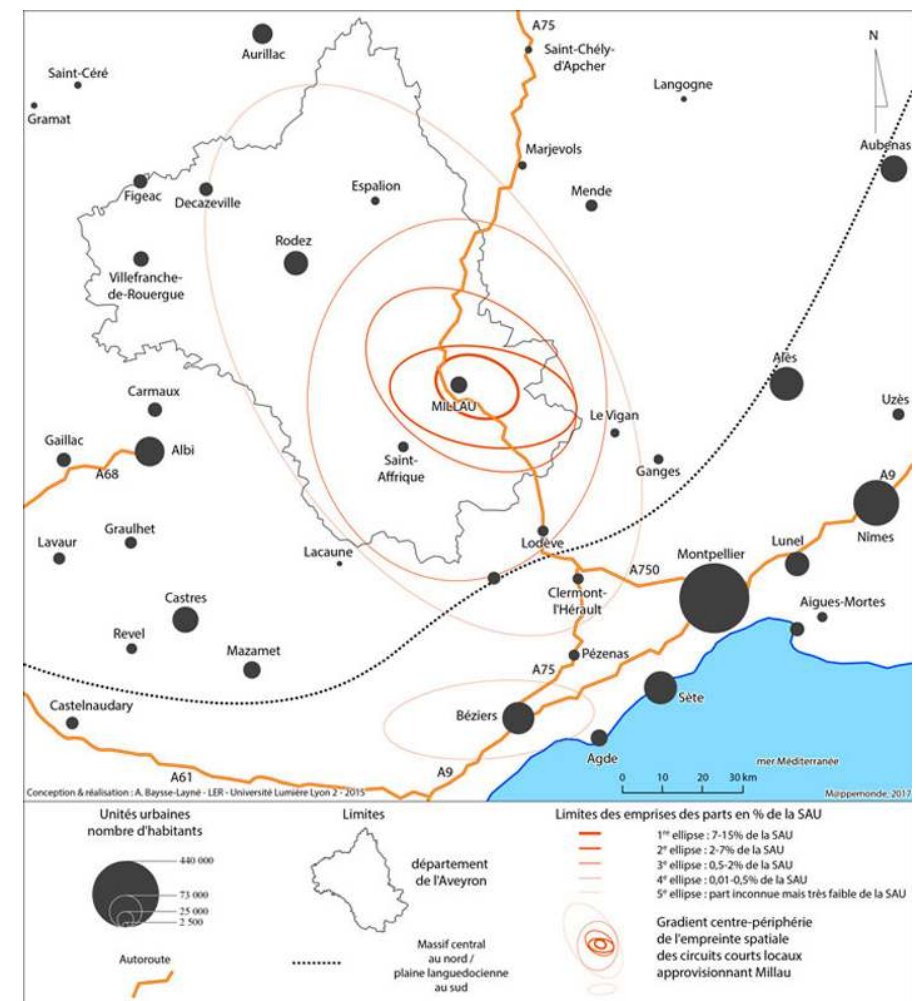

Conception \& réalisation : A. Baysse-Layné, LER - Université Lumière Lyon 2 - 2015 la première ellipse vers le sud, la deuxième vers le nord et la quatrième vers le sud et le 
nord. Nos entretiens ont toutefois surtout mis en avant le fait qu'elle facilite la commercialisation vers les villes du Languedoc.

Deux principaux écarts subsistent entre les formes théorique et empirique du premier secteur. La première ellipse présente une déformation positive vers l'ouest et le nord/ nord-est, qui correspond à la vallée du Tarn. Depuis 2010, six exploitations ont commencé à cultiver des terres en maraîchage biologique dans cette zone et vendent leur production à Millau dans au moins sept circuits courts. Cette zone a d'ailleurs été définie par la communauté de communes comme prioritaire pour sa politique de développement d'une filière maraîchère biologique.

Les quatre premières ellipses s'étendent particulièrement vers l'est et le sud-est. Ces zones ont des taux élevés d'exploitations en circuit court : $40 \%$ à $50 \%$ sur le Larzac ( figure 6c pour le repérage), $80 \%$ à $90 \%$ sur les premières pentes des Cévennes (Barry, 2012). Il serait intéressant d'intégrer ce taux d'exploitations en circuit court lors d'autres applications du modèle. Cette extension préférentielle vers les Causses pourrait également être en lien avec la dynamique spatiale du bassin de collecte de Roquefort. Centré sur le Larzac et ses marges occidentales dans les années 1840, ce bassin s'est étendu progressivement au reste des Causses, mais surtout au Saint-Affricain et au Tarn (Vabre, 2015) : en 1935, il se divisait à parts égales entre les Causses et les terres basses (Marres, 1935, p. 51-55, p. 122-138, p. 164). Actuellement, il se déplace vers l'ouest : très dense dans le Ségala, il l'est bien moins sur les hautes terres caussenardes (Frayssignes, 2007). Cette descente du bassin s'accompagne sur les Causses d'une croissance d'autres collectes de lait de brebis, mais des exploitations abandonnent aussi la production laitière. L'hypothèse selon laquelle la forte densité du premier secteur dans ces zones irait de pair avec leur sortie partielle du bassin de collecte de Roquefort reste toutefois à confirmer.

\section{Un approvisionnement en circuits longs principalement depuis des zones d'élevage aveyronnaises}

31 Les zones d'approvisionnement des intermédiaires des filières longues ont été délimitées, sans qu'un chiffrage intégral des surfaces agricoles de leurs fournisseurs ait pu être réalisé. Millau représente souvent un débouché marginal de ces unités (de $1 \%$ à $10 \%$ de leurs ventes) : leurs aires de collecte sont donc des zones de densité faible de l'empreinte alimentaire de la ville.

On retrouve autour de Millau les quatre types d'aires d'approvisionnement du modèle. Les plus fréquentes sont celles en gradient centre-périphérie, autour d'unités petites à moyennes (majoritairement des laiteries et fromageries). Au vu de nos données, il est difficile d'évaluer la pente de ces gradients: les aires apparaissent plutôt comme simplement continues ainsi que relativement circulaires et centrées sur l'atelier de transformation. Leurs limites sont soit celles d'un cercle dont le rayon est défini en distance ou en distance-temps, soit celles de petites régions agricoles ou naturelles. Huit exemples de ce type d'aire sont représentés sur la figure $\mathbf{6 a}$, dans des tons rouges, ocres et verts. Deux aires en tons bleus se rapprochent de ce type, à la seule exception près que l'unité de transformation est située hors de l'aire d'approvisionnement. 
Figure 6a. Exemples d'aires d'approvisionnement continues

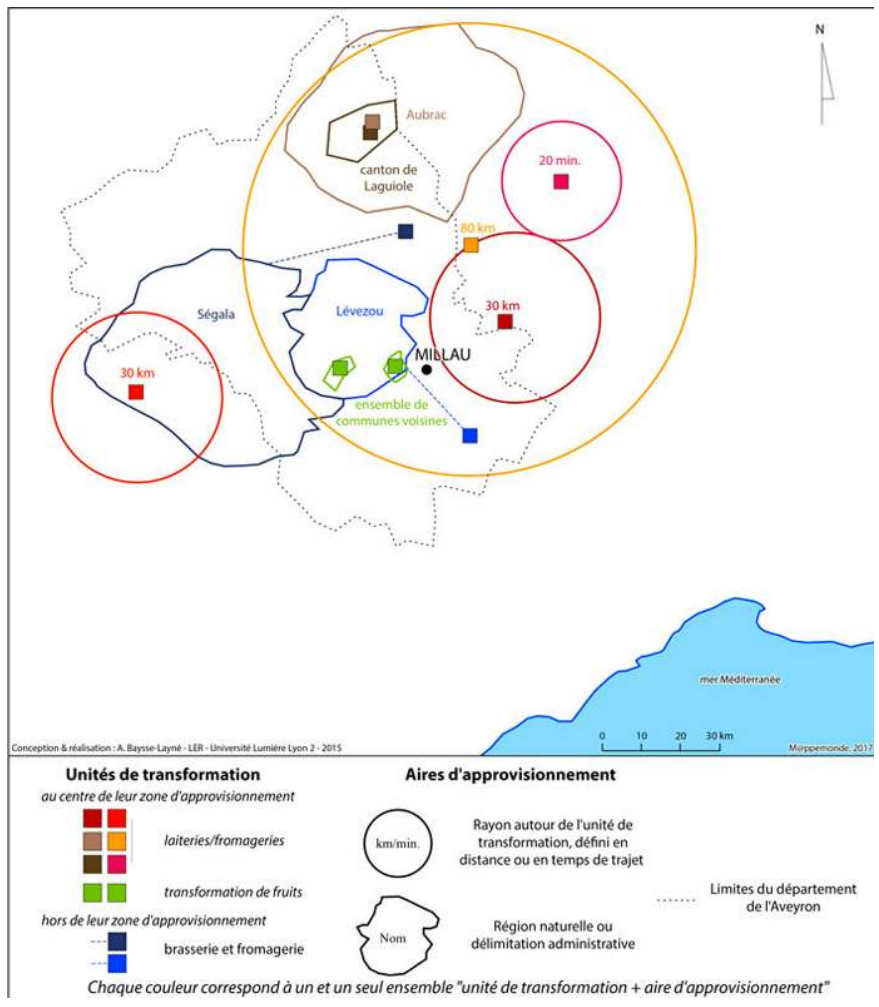

Conception \& réalisation : A. Baysse-Layné, LER - Université Lumière Lyon 2 - 2015

Les aires « complexes » sont également assez courantes, en majorité pour des unités de conserverie, charcuterie et viande fraîche. Elles reprennent principalement des limites de département, avec parfois l'adjonction de cantons ou d'un département voisin. La figure $\mathbf{6} \mathbf{b}$ donne l'exemple de onze de ces aires. 
Figure 6b. Exemples d'aires d'approvisionnement de formes complexes

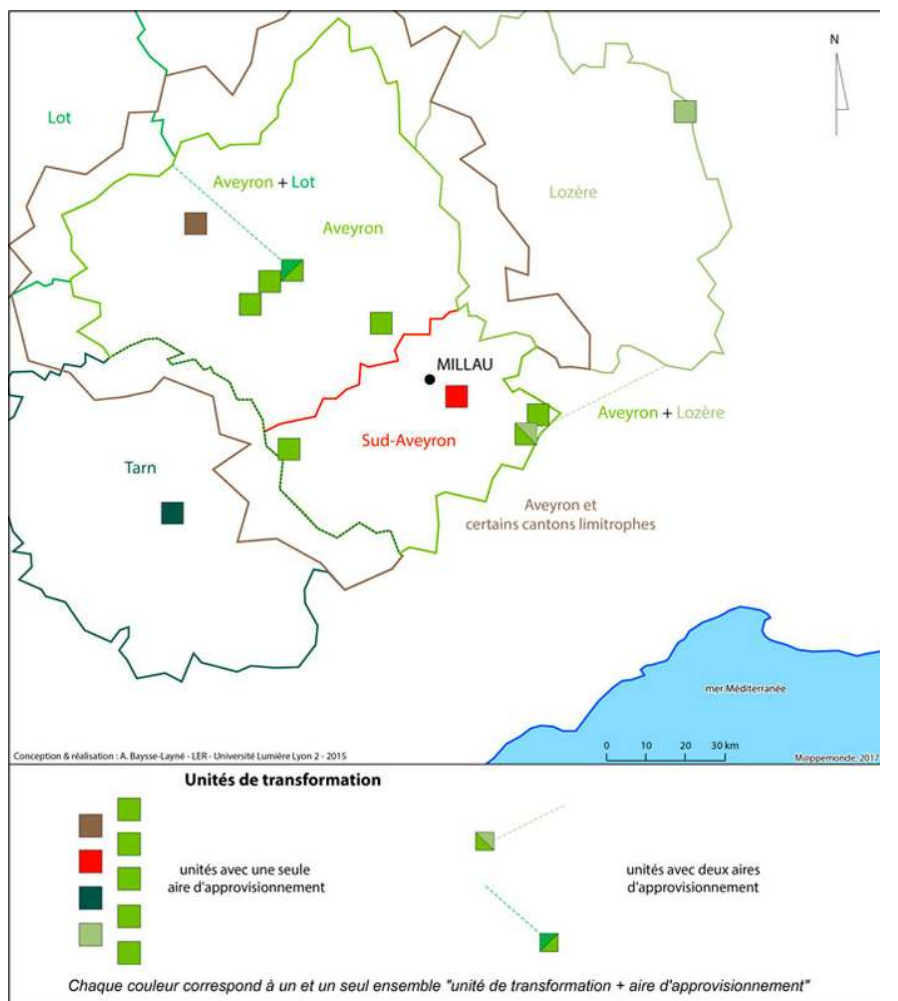

Conception \& réalisation : A. Baysse-Layné, LER - Université Lumière Lyon 2 - 2015

Les aires d'approvisionnement en archipel sont le fait de transformateurs laitiers petits à moyens, de boucheries et de minoteries. Pour le lait, elles comptent entre deux et quatre noyaux, les principaux étant le Lévezou et le Larzac. Les viandes proviennent surtout du Lévezou et de l'abattoir de Rodez, puis des Causses et de l'Aubrac, dans une moindre mesure du Ségala et d'autres abattoirs locaux. La distinction entre noyau central et noyaux secondaires n'apparaît pas. Cinq exemples de ces aires sont présentés sur la figure $6 \mathrm{c}$. 
Figure 6c. Exemples d'aires d'approvisionnement en archipel

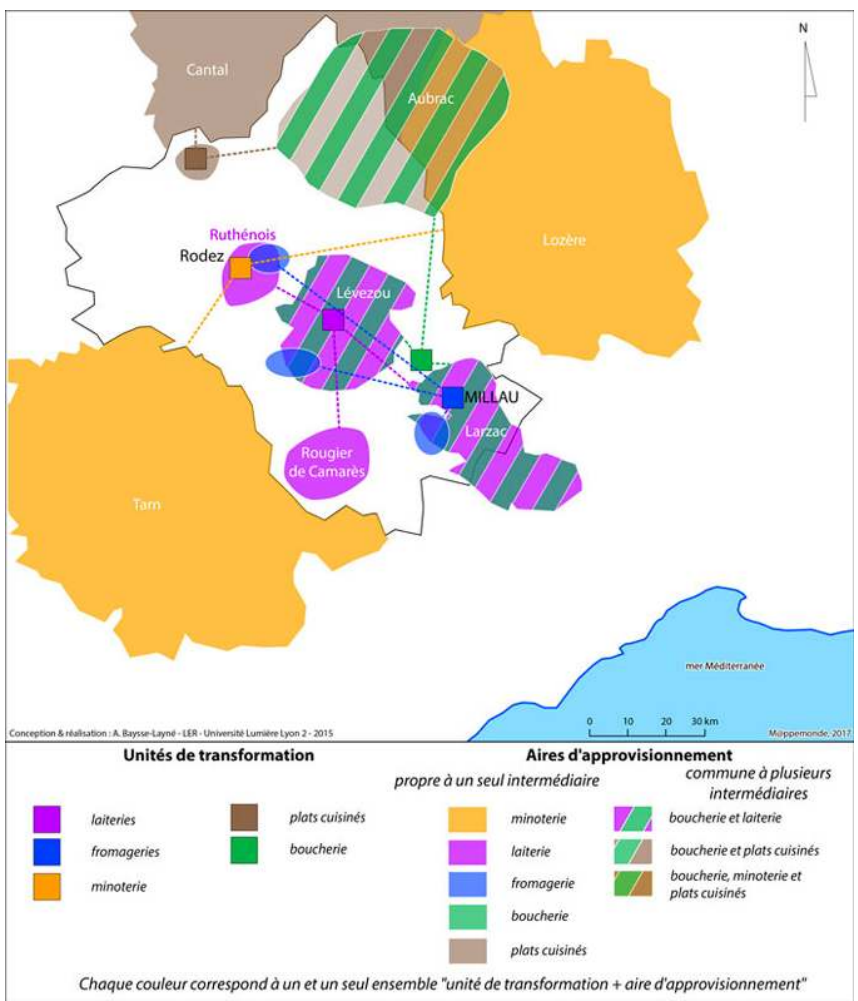

Conception \& réalisation : A. Baysse-Layné, LER - Université Lumière Lyon 2 - 2015 l'approvisionnement alimentaire local urbain. Pour ce faire, nous nous sommes appuyés sur les représentations et pratiques du «local» existant au sein de ces circuits de commercialisation, en considérant tous leurs acteurs (consommateurs, producteurs, intermédiaires) et en différenciant les logiques spatiales propres à chaque secteur de l'empreinte alimentaire. Une première application du modèle, à partir d'un recensement des terres approvisionnant localement la ville de Millau, confirme sa structure. 

gradient centre-périphérie qui s'étend préférentiellement dans l'entité administrative où se situe la ville et atteint des zones plus éloignées, mais aux orientations productives différentes. Ce gradient est également influencé par la forme du semis urbain, le maillage des axes de transport routier rapide et le taux d'exploitations en circuit court. Le deuxième secteur (empreinte spatiale des circuits longs) est la résultante d'aires d'approvisionnement des filières agroalimentaires, qui relèvent de cinq types : continue, circulaire et centrée sur l'infrastructure de transformation; continue, circulaire avec infrastructure de transformation hors du bassin de collecte; en archipel; à forme complexe ; en maillage du territoire. Les données - partielles - que nous avons récoltées concernant Millau montrent que l'empreinte alimentaire locale de la ville est particulièrement dense en Aveyron et dans les zones montagneuses à l'est et au sud alors qu'elle est plus restreinte à proximité de Saint-Affrique. Elle s'étend, certes faiblement, jusque dans la plaine languedocienne, et en partie le long de l'A75.

Notre étude confirme la fluidité des échelles et des définitions du « local », qui apparaît comme une catégorie à la fois continue et discontinue, graduelle et territoriale. Si les conceptions des consommateurs à cet endroit ont bien été décrites par la littérature, un manque relatif apparaît quant aux critères mobilisés par les producteurs pour définir l'extension spatiale d'une commercialisation locale de leurs produits. Ce manque est plus profond concernant les approvisionnements en intrants, qui - à notre connaissance n'ont fait l'objet d'aucune étude géographique. La recherche a également peu abordé la question de la forme spatiale des bassins de collecte des filières agroalimentaires longues.

Plusieurs pistes s'ouvrent au terme de ce travail. Le modèle graphique de l'empreinte spatiale de l'approvisionnement alimentaire local urbain reste à confronter à d'autres cas que celui de Millau. Des applications à des villes de tailles variées et situées dans des régions agricoles aux degrés de spécialisation différents permettraient de mieux cerner sa validité. La récolte de données relatives à la provenance des intrants constitue un préalable à une modélisation du troisième secteur. En considérant les amendements, les aliments pour le bétail, les semences et les produits phytosanitaires, une telle démarche complèterait le tableau de l'empreinte alimentaire urbaine. Enfin, il reste à définir une démarche permettant la cartographie de la densité cumulée des trois secteurs de l'empreinte alimentaire locale.

\section{BIBLIOGRAPHIE}

ADAMS D. C., ADAMS A. E. (2011). “De-placing local at the farmer's market: Consumer conceptions of local foods". Journal of Rural Social Sciences, vol. 26, n² 2, p. 74-100.

BARLES S. (2008). « Comprendre et maîtriser le métabolisme urbain et l'empreinte environnementale des villes ». Responsabilité et environnement, ${ }^{\circ}$ 52, p. 21-26.

BARRY C. (2012). « Un producteur sur cinq vend en circuit court ». Agreste Primeur, n² 275, p. 1-4. 
BECKER H. (1998). Allgemeine historische Agrargeographie. Stuttgart : Teubner, coll.

«Studienbücher », 333 p. ISBN 3-519-03439-5

BILLEN G., BARLES S., CHATZIMPIROS P., GARNIER J. (2012). “Grain, meat and vegetables to feed Paris: where did and do they come from? Localising Paris food supply areas from the eighteenth to the twenty-first century". Regional Environmental Change, vol. 12, n² 2, p. 325-335.

BLANQUART C., GONÇALVES A. (2011). La diversité de l'inscription spatiale des circuits courts. Colloque Migrations et territoires, ASRDLF, Schœlcher (Martinique), 6-8 juillet 2011.

BROWN C. (2003). “Consumer's preferences for locally produced food: A study in southeast Missouri". American Journal of Alternative Agriculture, vol. 18, n 4, p. 213-224.

CAPT D., LEPICIER D., LESEIGNEUR A. (2014). « Le rôle des territoires de projets infra-régionaux sur l'agriculture et l'alimentation. Le cas du développement de circuits de proximité ». Géocarrefour, vol. 89, $\mathrm{n}^{\circ} 1-2$, p. 105-113.

CARROLL B. E., FAHY F. (2014). "Locating the locale of local food: The importance of context, space and social relations". Renewable Agriculture and Food Systems, FirstView, 14 p.

CHATZIMPIROS P. (2011). Les empreintes environnementales de l'approvisionnement alimentaire: Paris, ses viandes et lait, $\mathrm{XIX}^{e}-\mathrm{XX} \mathrm{X}^{e}$ siècles. Marne-la-Vallée : Université Paris-Est, thèse de doctorat d'aménagement de l'espace et d'urbanisme, $351 \mathrm{p}$.

CHEVALLIER M., DELLIER J., PLUMECOCQ G., RICHARD F. (2014). « Dynamiques et structuration des circuits courts agroalimentaires en Limousin : distance institutionnelle, proximités spatiale et relationnelle ». Géographie, économie, société, vol. 16, n 3, p. 339-362.

CORNIAUX C., BARITAUX V., MADELRIEUX S. (2014). Entre (re-)localisation et globalisation : analyse des stratégies spatiales des laiteries dans six bassins laitiers. $8^{\text {es }}$ journées de recherche en sciences sociales - SFER, Grenoble, 11-12 déc. 2014.

CORSI S., MAZZOCCHI C., SALI G., MONACO F., WASCHER D. (2015). « L'analyse des systèmes alimentaires locaux des grandes métropoles. Proposition méthodologique à partir des cas de Milan et de Paris ». Cahiers Agricultures, vol. 24, n 1, p. 28-36.

DARROT C. (dir.) (2012). Rennes Métropole, ville vivrière (2) ? Rennes : Agro Campus Ouest, Projet Ingénieur, $177 \mathrm{p}$.

DELFOSSE C. (2013). « Produits de terroir et territoires. Des riches heures du développement rural à la gouvernance métropolitaine ». Sud-Ouest européen, $\mathrm{n}^{\circ}$ 35, p. 17-29.

DELFOSSE C. (2012). « Conclusion générale. Point de vue d'une géographe ». In PRIGENT-SIMONIN A. H., HÉRAULT-FOURNIER C. (coord.), Au plus près de l'assiette. Pérenniser les circuits courts. Versailles : Quæ, coll. « Sciences en partage », p. 233-236. ISBN 978-2-75921-780-9

FELDMANN C., HAMM U. (2015). “Consumers' perceptions and preferences for local food: A review”. Food Quality and Preference, vol. 40-A, p. 152-164.

FRAYSSIGNES J. (2007). « L'AOC Roquefort : une filière emblématique ». MediTERRA, Identités et qualité des produits alimentaires méditerranéens, p. 147-184.

FUTAMURA T. (2007). "Made in Kentucky: The meaning of 'local' food products in Kentucky's farmers' markets". The Japanese Journal of American Studies, nº 18, p. 209-227.

GALLONS J., TOENSMEYER U.C., BACON J.R, GERMAN C.L. (1997). “An analysis of consumer characteristics concerning direct marketing of fresh produce in Delaware: A case study". Journal of Food Distribution Research, vol. 28, n 1, p. 98-106. 
GARÇON L. (2012). « La mobilisation d'acteurs autour de biens ordinaires requalifiés : un exemple en Ligurie ». POUR, $\mathrm{n}^{\circ}$ 215-216, p. 161-168.

HU W., BATTE M. T., WOODS T., ERNST S. (2010). What is local and for what foods does it matter? $42^{\text {nd }}$ Southern agricultural economics association annual meeting, Orlando, 6-9 février 2010, 37 p. ILBERY B., WATTS D., SIMPSON S., GILG A., LITTLE J. (2006). "Mapping local foods: evidence from two English regions". British Food Journal, vol. 108, n³ 3, p. 213-225.

JÉNOT F., DESMAISON P. (2009). Historique et stratégies des entreprises laitières de Charentes-Poitou pour la production de fromage de chèvre, entre logiques de filière et de territoire. Actes des $16^{\text {es }}$ journées $3 \mathrm{R}-$ Rencontres Recherche Ruminants (INRA-Institut de l'élevage), Paris, 2-3 déc. 2009, p. 325-328. KAHN F., PRIOR C. (2010). "Evaluating the urban consumer with regard to sourcing local food: a Heart of England study". International Journal of Consumer Studies, vol. 34, n² 2, p. 161-168.

KRENZLIN A. (1962). Die Entwicklung der Gewannflur als Spiegel kulturlandschaftlicher Vorgänge. Verhandlungen des 33. Deutschen Geographentages, Cologne, 22-26 mai 1961, p. 305-322.

LIENAU C. (1995). Die Siedlungen des ländlichen Räumes. Braunschweig : Westermann, coll. "Das geographische Seminar", 246 p. ISBN 978-3141602838

MCENTEE J. (2010). “Contemporary and traditional localism: a conceptualisation of rural local food”. Local Environment, vol. 15, n 9-10, p. 785-803.

MARGETIC C. (2014). « Des industries agroalimentaires françaises face à des enjeux majeurs ». L'Information géographique, vol. 78, $\mathrm{n}^{\circ} 4$, p. 27-47.

MARIE M., BERMOND M., MADELINE P., COINAUD C. (2014). « Une typologie des combinaisons d'utilisation agricole du sol en France en 2010 : propositions méthodologiques ». M@ppemonde, n - 114 (2-2014). En ligne : http://mappemonde-archive.mgm.fr/num42/articles/art14203.html MARRES P. (1935). Les Grands Causses : étude de géographie physique et humaine. Tours : Arrault, 446 p. MARTY P. (2013). Les appropriations urbaines de la question agricole. Le cas de Brive de 1945 à 2012. Paris : Université Paris 1-Panthéon-Sorbonne, thèse de doctorat de géographie aménagement, $609 \mathrm{p}$. MÜLLER-WILLE W. (1955). Agrarbäuerliche Landschaftstypen in Nordwestdeutschland. Verhandlungen des 29. Deutschen Geographentages, Essen, 25-30 mai 1953, p. 179-186.

ostrom M. (2006). “Everyday meanings of 'Local Food': Views from Home and Field”. Community development, vol. $37, \mathrm{n}^{\circ} 1$, p. 65-78.

PENKER M. (2006). "Mapping and measuring the ecological embeddedness of food supply chains". Geoforum, vol. 37, n³, p. 368-379.

PETERS C. J., BILLS N. L., LEMBO A. J., WILKINS J. L., FICK G. W. (2009). "Mapping potential foodsheds in New York State: A spatial model for evaluating the capacity to localize food production". Renewable Agriculture and Food Systems, vol. 24, $\mathrm{n}^{\circ}$ 1, p. 72-84.

PETIT C. (2013). Transitions des exploitations agricoles vers l'agriculture biologique dans un territoire approche par les interactions entre systèmes techniques et de commercialisation. Application aux aires d'alimentation de captages en Île-de-France. Paris : AgroParisTech, thèse de doctorat d'agronomie, $412 \mathrm{p}$.

PETIT C., AUBRY C. (2012). Géographie de l'agriculture biologique et opportunités locales de débouchés commerciaux pour les exploitations agricoles. Cas de la luzerne en Île-de-France. Journée technique. La luzerne, incontournable en grandes cultures biologiques? ITAB-Arvalis Institut du végétal, Villarceaux, 13 juin 2012, poster. 
POULOT M. (2010). «L'agriculture francilienne dans la seconde moitié du XX $\mathrm{XX}^{\mathrm{e}}$ siècle : vers un postproductivisme de proximité ?». POUR, n² 205-206, p. 161-177.

POULOT M. (2014). « Histoires d'AMAP franciliennes : quand manger met le local dans tous ses états ». Territoire en mouvement. Revue de géographie et aménagement, $\mathrm{n}^{\circ} 22$, p. 40-53.

PRALY C., CHAZOULE C., DELFOSSE C., MUNDLER P. (2014). « Les circuits de proximité, cadre d'analyse de la relocalisation des circuits alimentaires ». Géographie, économie, société, vol. 16, n 4, p. 455-478.

SCHEFFER S., DALIDO A.-L. (2010). Observer les circuits courts à l'échelle d'un territoire : proposition d'un modèle d'analyse spatiale des données, en termes de systèmes d'information géographique. International EAAE-SYAL Seminar - Spatial Dynamics in Agri-food Systems, Parme, 27-30 octobre 2010, 18 p.

SCHNELLER M. (2010). Une approche géographique des circuits courts de fruits et légumes : étude des espaces de la distribution et propositions d'action en Languedoc-Roussillon. Montpellier : Université Paul Valéry Montpellier 3, mémoire de master 2 (Innovation et Développement des Territoires Ruraux), $161 \mathrm{p}$.

SELFA T., QAZI J. (2005). "Place, taste, or face-to-face? Understanding producer-consumer networks in 'local' food systems in Washington State”. Agriculture and Human Values, n² 22, p. 452-464.

VABRE S. (2015). Le sacre du roquefort : l'essor d'une industrie agro-alimentaire (fin XVIII $\left.{ }^{e}-1925\right)$. Rennes : PUR, coll. « Tables des Hommes », 584 p. ISBN 978-2-7535-3557-2

VAUDOIS J. (2000). « Les dynamiques spatiales des productions légumières : l'évolution récente des bassins endiviers de Nord-Picardie ». Méditerranée, vol. 95, n 3, p. 65-74.

VON THÜNEN J. H. (1826). Der isolierte Staat in Beziehung auf Landwirtschaft und Nationalökonomie. Hambourg : Perthes, 290 p.

WASCHER D., ZASADA I., JEURISSEN L., ARCINIEGAS G., DE KROES J., SALI G., CORSI S., MONACO F., SCHMUTZ U., GLAVAN M., POHLE D., VAN EUPEN M. (2015). Indicators, Tools and Method for Metropolitan Footprint Tools. Délivrable D3.3 du projet de recherche "Foodmetres: Food Planning and Innovation for Sustainable Metropolitan Regions". En ligne : http://www.foodmetres.eu/

WILKINS J.L., BOKAER-SMITH J.C., HILCHEY D. (1996). Local Foods and Local Agriculture: A Survey of Attitudes among Northeastern Consumers. Rapport d'étude, Northeast Regional Food Guide Project, Cornell University, Division of Nutritional Sciences.

\section{NOTES}

1. Code présent sur tout produit alimentaire transformé, quand le nom et l'adresse de l'entreprise qui l'a préparé ne sont pas précisés sur l'emballage. Il se compose du numéro du département, suivi du numéro INSEE de la commune et du numéro de l'entreprise dans cette commune. Ex : 12.203.018 correspond à l'entreprise Société (018), située à Roquefort-sur-Soulzon (203), dans l'Aveyron (12).

2. http://transitionculture.org/2009/03/09/foodzoning-the-foodshed/

3. https://www.colibris-lemouvement.org/passer-a-laction/creer-son-projet/developper-uneceinture-maraichere-autour-villes

4. http://www.ladepeche.fr/article/2009/10/16/695054-une-ceinture-maraichere-autour-derodez.html

5. https://web.archive.org/web/20130823155621/http://www.iledefrance.fr/fil-actus-region/ recreer-ceinture-agricole-autour-paris 
6. Comme par exemple «Terres d'ici. Agriculteurs de l'Y Grenoblois » ou «Les jardins du Pays d'Aubagne ».

\section{RÉSUMÉS}

Cet article propose de modéliser graphiquement la morphologie des espaces agricoles qui «nourrissent» les villes par l'intermédiaire de circuits locaux. L'assise spatiale de ces approvisionnements est divisée en trois secteurs, dont les formes sont évaluées à partir d'une étude bibliographique. L'application du modèle au cas de Millau (Aveyron) montre que l'empreinte spatiale de l'alimentation locale urbaine s'étend suivant deux types de logiques: celles relatives à la définition du «local » par les consommateurs et les producteurs et celles propres aux industries agroalimentaires.

This article aims to establish a graphic model of agricultural spaces that "feed" cities through local supply chains. The spatial footprint of local foodsheds is divided into three sectors and their morphology is assessed using existing scholarship. The model is applied to the case study of Millau, a small, French town located in a highland region. Our study highlights two factors that shape the spatial footprints of urban local food supplies. 1) the way consumers and producers define "local" 2) the dynamics of agro industry.

Ese artículo propone modelizar gráficamente la morfología de los espacios agropecuarios que aprovisionan a las ciudades mediante circuitos locales de kilómetro cero. La base territorial de estos espacios de abastecimiento están divididos en tres sectores, cuyas morfologías son evaluadas mediante una revisión bibliográfica. El modelo se ha aplicado a la pequeña ciudad francesa de Millau, ubicada en una zona montañosa. Los resultados identifican dos factores que explican la forma de la huella espacial de la alimentación local de esta ciudad: 1) la consideración y prácticas de lo que consideran como "local" consumidores y productores, 2) las dinámicas propias de la agroindustria.

\section{INDEX}

Mots-clés : approvisionnement alimentaire, définitions du « local », empreinte alimentaire urbaine, Millau, modélisation spatiale

Keywords : definitions of "local", food supply, Millau, spatial modeling, urban foodshed

Palabras claves : abastecimiento alimentario, huella alimentaria urbana, definiciones del "local", Millau, modelado espacial

\section{AUTEUR}

\section{ADRIEN BAYSSE-LAINÉ}

Doctorant en géographie, chargé d'enseignement Université Lumière Lyon 2/INRA Montpellier 